\title{
Pork Peptone Stimulates Cholecystokinin Secretion from Enteroendocrine Cells and Suppresses Appetite in Rats
}

\author{
MKNB Sufian, Tohru Hira, Kyoko Miyashita, Takashi Nishi, Kozo Asano, and Hiroshi Hara ${ }^{\dagger}$ \\ Division of Applied Bioscience, Graduate School of Agriculture, Hokkaido University, \\ Kita-9, Nishi-9, Kita-Ku, Sapporo 060-8589, Japan
}

Received January 24, 2006; Accepted April 12, 2006; Online Publication, August 23, 2006

[doi:10.1271/bbb.60046]

We found that soybean $\beta$-conglycinin peptone (BconP) suppresses food intake through cholecystokinin (CCK) release from enteroendocrine cells in association with binding of the peptone to rat small intestinal brush border membrane (BBM). The aim of the present study was to find new appetite suppressing peptides. Peptones from chicken, pork, beef, beef liver, and egg white were examined for activities to bind with rat BBM, CCKrelease from enteroendocrine cell line STC-1, and induce satiety in rats. Chicken and pork peptone (ChickP and PorkP) bound to BBM with highest ability as evaluated with a surface plasmon biosensor. PorkP and ChickP released CCK in higher amounts than BconP from STC-1 cells dose-dependently, with highest stimulation by PorkP. An orogastric preload of PorkP, but not ChickP, suppressed food intake similarly to BconP, dose-dependently. These results suggest that PorkP interacts directly with the small intestinal CCK cells to release $\mathrm{CCK}$, and that it suppresses appetite in rats.

Key words: cholecystokinin; pork peptone; brush-border membrane; food intake; STC-1 cells

Excess food intake is a prominent contributor to several habit-related diseases, e.g., obesity and diabetes. Hence control of appetite has the merit of preventing increasing threats from these medical complications. In this perspective, the development of anorectic food components is helpful towards a healthy life.

The gut-brain satiety hormone cholecystokinin (CCK) is secreted by the enteroendocrine cells (I cells) upon ingestion of nutrients into the small intestine. ${ }^{1)}$ The role of $\mathrm{CCK}$ as a major endocrine determinant in appetite control is well-documented.,3) The role of peripheral CCK in satiety is evident from studies with CCK receptor antagonists and vagotomized subjects, which have been shown to abolish anorexia produced by gastric or duodenal delivery of nutrients. ${ }^{4,5)}$
In rats, dietary proteins have been reported to be more satiating than carbohydrate or fat, ${ }^{6)}$ and to be a major stimulus of intestinal CCK release. ${ }^{7,8)}$ Endogenous CCK has been shown to induce anorexia after gastric or duodenal delivery of peptones. ${ }^{4,5)}$ It is believed that CCK release by dietary protein is regulated by endogenous, trypsin-sensitive CCK-releasing peptides, ${ }^{9)}$ but some studies both in vivo and in vitro have found that dietary protein and its peptide react on CCK-producing cells directly to stimulate CCK release. ${ }^{10-12)}$ Previously we found that intraduodenal infusion of a peptic hydrolysate of soybean $\beta$-conglycinin, $\beta$-conglycinin peptone $(\mathrm{BconP})$, suppresses food intake through this CCK release. ${ }^{13,14)}$

It has been suggested that binding of dietary proteins or peptides to the rat small intestinal brush-border membrane $(\mathrm{BBM})$ is an integral part of a common sensory mechanism to release CCK. $\left.{ }^{15}\right) \mathrm{We}$ found that appetite suppression by BconP corresponds to the binding ability of this peptone to the rat small intestinal BBM as well as stimulation of CCK release directly from rat isolated dispersed intestinal mucosal cells. ${ }^{14)}$

Cellular preparations from rat isolated dispersed intestinal mucosa might not consist solely of CCKproducing I-cells due to cell heterogeneity in the intestinal mucosa. Isolation of native intestinal I-cell remains a tedious process, and it produced fairly low yields. ${ }^{16)}$ An enteroendocrine cell line, STC-1 cells have been shown to be a suitable model for studying CCK release, which responds to several physiological stimulants including nutrients, hormones, and luminal CCKreleasing factors. ${ }^{11,12,17,18)}$

The purpose of the present study was to find new appetite suppressing peptides from dietary protein sources, other than $\beta$-conglycinin, that involve with CCK release. The effects of dietary proteins derived from animal sources on CCK release have not yet been studied extensively by in vitro or in vivo models. Hence we prepared peptic hydrolysates from some commonly

\footnotetext{
$\dagger$ To whom correspondence should be addressed. Tel: +81-11-706-3352; Fax: +81-11-706-2504; E-mail: hara@chem.agr.hokudai.ac.jp

Abbreviations: BBM, brush-border membrane; BconP, $\beta$-conglycinin peptone; BeefP, beef peptone; BLP, beef liver peptone; BSA, bovine serum albumin; CCK, cholecystokinin; ChickP, chicken peptone; EWP, egg white peptone; PorkP, pork peptone; RU, resonance unit
} 
edible animal proteins, and first examined their ability to bind with the rat small intestinal BBM using a surface plasmon resonance-biosensor. The peptones with the highest binding affinity were assessed as to whether they stimulated CCK release from the murine enteroendocrine cell line STC-1, and then it was determined whether they induce satiety in rats by food intake studies following orogastric preload.

\section{Materials and Methods}

Materials. Materials used were obtained as follows: Beef thigh meat, beef liver, pork thigh meat, chicken breast meat, and eggs were purchased from commercial sources. Pepsin and BSA (bovine serum albumin) were purchased from Sigma Chemical (St. Louis, MO), while soybean $\beta$-conglycinin was a gift from Fuji Oil (Osaka, Japan). Protein fractions were prepared from these animal meats, liver, and egg white. Briefly, meat, liver, and egg white was boiled in water to inactivate endogenous enzymes and homogenized in chloroformmethanol (2:1) to extract lipids. After chloroform extraction, the residue was washed with ethanol, airdried, and milled.

Animals and peptide preparations. Male SpragueDawley rats (Japan SLC, Hamamatsu, Japan), weighing about $270-370 \mathrm{~g}$, were kept in individual stainless steel cages in a temperature-controlled room maintained at $23 \pm 2{ }^{\circ} \mathrm{C}$ with a 12-h light-dark cycle (0800-2000 light period), rats were fed a casein-based basal stock diet (Table 1) during the acclimation period. The Hokkaido University Animal Committee approved the study, and the animals were maintained in accordance with the guidelines for the care and use of laboratory animals of Hokkaido University.

Hydrolysis of protein by pepsin was performed as previously described, with slight modifications. ${ }^{19)}$ Briefly, chicken, pork, beef, beef liver, egg white, and $\beta$ conglycinin were suspended in phosphate buffer and homogenized with a Polytron homogenizer (Kinematica, Lucerne, Switzerland). Then these ingredients were treated with pepsin $(10,870$ units/g substrate) at $\mathrm{pH} 1.8$

Table 1. Composition of Basal Stock Diet

\begin{tabular}{lc}
\hline \hline & $\mathrm{g} / \mathrm{kg}$ diet \\
\hline Casein $^{\mathrm{a}}$ & 250 \\
Corn oil & 50 \\
Mineral mixture $^{\mathrm{b}}$ & 35 \\
Vitamin mixture $^{\mathrm{b}}$ & 10 \\
Choline bitartrate $^{\text {Cellulose }}$ & 2.5 \\
Sucrose $^{\mathrm{c}}$ & 50 \\
\end{tabular}

${ }^{a}$ Lactic casein (ALACID; New Zealand Dairy Board, Wellington, New Zealand).

${ }^{\mathrm{b}}$ The mineral and vitamin mixtures were prepared in accordance with the AIN-93G formulation. ${ }^{23)}$

${ }^{\mathrm{c} C}$ Crystallized cellulose (AVICEL; Asahi Chemical Industry, Tokyo). and $37^{\circ} \mathrm{C}$ for $10 \mathrm{~min}$, followed by neutralization, desalting, and lyophilization. The peptones obtained were ChickP (chicken peptone), PorkP (pork peptone), BeefP (beef peptone), BLP (beef liver peptone), EWP (egg white peptone), and $\mathrm{BconP}$ ( $\beta$-conglycinin peptone) respectively. The protein contents of these prepared peptones was determined using Lowry's protein assay, ${ }^{20)}$ by which $104,100,103,79,96$, and $86 \%$ purity was observed for ChickP, PorkP, BeefP, BLP, EWP, and $\mathrm{B}$ conP respectively against $\mathrm{BSA}$ as standard.

Binding of test peptones to rat small intestinal brush border membranes. The binding ability of the test peptones to the rat small intestinal BBM was estimated using a surface plasmon resonance-biosensor (Biacore 3000 system, Biacore AB, Uppsala, Sweden) and Sensor chip CM5, as previously described. ${ }^{19)}$ Briefly, the soluble components of rat intestinal BBM were prepared by solubilization with Triton X-100; they were immobilized as ligand over a flow cell surface (a test flow cell) of the CM5 sensor chip by an amine-coupling procedure. ${ }^{15)}$ Ethanolamine was immobilized onto another flow cell as a reference. Test peptone was dissolved $(100 \mu \mathrm{g} / \mathrm{ml})$ in HBS-E buffer containing $10 \mathrm{~mm}$ HEPES, $150 \mathrm{mmol} / 1 \mathrm{NaCl}$, and $3 \mathrm{mmol} / 1$ EDTA, $\mathrm{pH} \mathrm{7.4}$, and injected over both the test and reference flow cells at a rate of $10 \mu \mathrm{l} / \mathrm{min}$ for $2 \mathrm{~min}$ as the analyte. The amount of protein bound to the immobilized BBM proteins on the sensor chip was represented in resonance units (RU).

CCK secretion study from enteroendocrine cells. STC-1 cells were kindly provided by Dr. D. Hanahan (University of California, San Francisco). These cells were originally derived from an intestinal endocrine tumor obtained from double transgenic mice. ${ }^{21)}$ STC-1 cells were grown in Dulbecco's modified Eagle's Medium (DMEM; $4.5 \mathrm{~g} / 1$ glucose, with L-glutamin, without sodium pyruvate; Gibco BRL 12100-038, Grand Island, NY) containing $10 \%$ fetal calf serum, $100 \mathrm{U} / \mathrm{ml}$ penicillin, and $100 \mu \mathrm{g} / \mathrm{ml}$ streptomycin. A humidified $5 \% \mathrm{CO}_{2}$ atmosphere was maintained at $37^{\circ} \mathrm{C}$. Cells between passages 30 and 40 were used at $80-90 \%$ confluence.

For secretion studies, $1.25 \times 10^{5}$ cells were seeded into 48-well plates and used when they reached subconfluence after a culture for 2-3d. The cultured cells were then washed twice with HEPES buffer $(140 \mathrm{~mm}$ $\mathrm{NaCl}, 4.5 \mathrm{~mm} \mathrm{KCl}, 20 \mathrm{~mm}$ HEPES, $1.2 \mathrm{~mm} \mathrm{CaCl}_{2}, 1.2$ $\mathrm{mM} \mathrm{MgCl}_{2}, 10 \mathrm{~mm}$ Glucose, $\mathrm{pH}$ 7.4), and test peptones of each concentration $(1,2$, and $5 \mathrm{mg} / \mathrm{ml})$ dissolved in HEPES buffer were added to the wells. After a 60-min incubation at $37^{\circ} \mathrm{C}$ in the $\mathrm{CO}_{2}$ incubator, medium was collected on ice and centrifuged at $850 \times g$ at $4{ }^{\circ} \mathrm{C}$ for $5 \mathrm{~min}$ to remove cells, and the supernatant was stored at $-30{ }^{\circ} \mathrm{C}$ until CCK measurement. The CCK released was measured with an enzyme immunoassay kit (Phoenix Pharmaceuticals, Belmont, CA). 
Experimental protocol for food intake studies. Rats were allowed an acclimation period of about $7 \mathrm{~d}$ to adapt to the new environment and a semi-purified diet (AIN93G), and were trained daily by an orogastric procedure with distilled water using a gastric tube (Safeed Feeding Tube Fr. 5, $40 \mathrm{~cm}$; Terumo, Tokyo). Experiments with test peptides were done after obtaining a stable daily food intake with the orogastric procedure in water in this period.

After $24 \mathrm{~h}$ of food deprivation, rats were administered the test peptone preloads $(10-50 \mathrm{mg} / 1 \mathrm{ml}$ distilled water) by the orogastric method. Thirty minutes after peptide administration, the rats were given $25 \mathrm{~g}$ of basal stock diet (Table 1) for $60 \mathrm{~min}$, and the remaining and spilled diet was collected and weighed. The experiment was conducted until every rat received each preload once. The rats were used twice a week for the experiments. Water and BconP preloads were administered as negative and positive controls respectively.

Statistical analyses. All results are expressed as means \pm SEM. Food intake after the orogastric preload of each peptone was compared separately with the water preload as control using Student's paired $t$-test $(P<$ 0.05). The results of the CCK secretion study were expressed as net increases in released CCK (pM) from STC-1 cells, and were analyzed by Duncan's multiple range test.

\section{Results}

Binding of the test peptones to the rat intestinal brush border membrane components

Among the tested peptones, ChickP, PorkP, and BeefP similarly formed higher bindings at a concentration of $100 \mu \mathrm{g} / \mathrm{ml}$ to the solubilized BBM of rat small intestine than other peptones (Table 2). Conversely, a moderate binding ability was shown by BLP (beef liver peptone), while EWP (egg white peptone) showed much less binding than the other peptones tested.

$C C K$ releasing activity of PorkP and ChickP from the enteroendocrine cell line STC-1

The results of the CCK-releasing activity of PorkP and ChickP after their exposure to the CCK-producing

Table 2. Binding Response of Prepared Test Peptones to the Soluble Components of Rat Small Intestinal Brush Border Membrane

\begin{tabular}{lcc}
\hline \hline Source & Peptone & Binding $\left(\mathrm{RU}^{\mathrm{a}}\right)$ \\
\hline Chicken & ChickP & $390 \pm 23$ \\
Pork & PorkP & $355 \pm 20$ \\
Beef & BeefP & $346 \pm 79$ \\
Beef liver & BLP & $194 \pm 12$ \\
Egg white & EWP & $38 \pm 6$ \\
\hline
\end{tabular}

${ }^{\mathrm{a}} \mathrm{RU}$, resonance unit. Values are means $\pm \mathrm{SEM}$ of three injections per peptone on the rat small intestinal brush-border membrane-immobilized sensor chip.

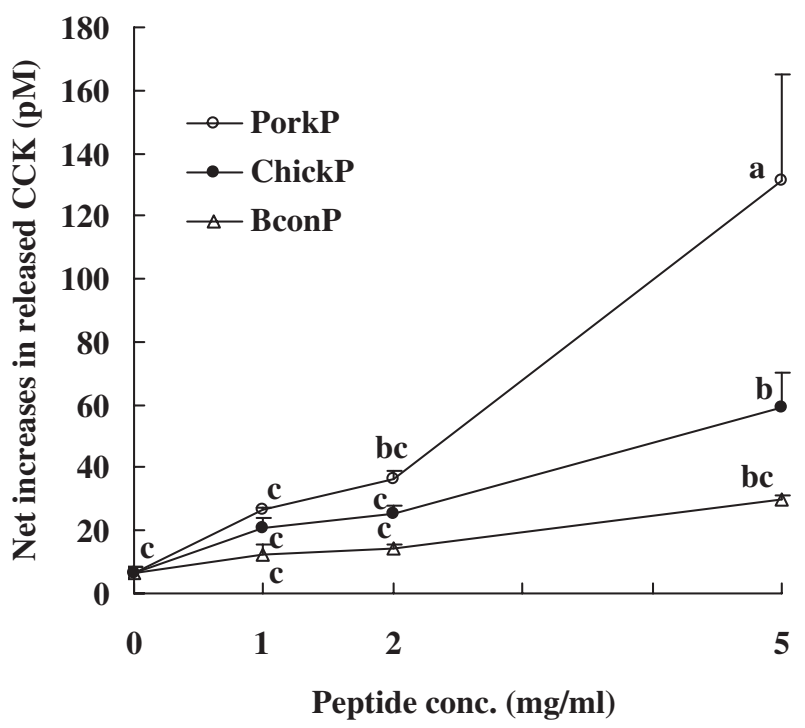

Fig. 1. CCK Released from Enteroendocrine STC-1 Cells after Exposure to PorkP (Pork peptone), ChickP (Chicken peptone), and BconP ( $\beta$-conglycinin peptone) at 1,2 , and $5 \mathrm{mg} / \mathrm{ml}$ Concentrations for 60 min Relative to the Blank.

Values are means \pm SEM of three repeated measurements. Means without a common letter are significantly different $(P<0.05)$.

cell line STC-1 are presented in Fig. 1. PorkP and ChickP stimulated CCK release at higher levels than BconP at each concentration in a dose-dependent fashion. A greater stimulation of CCK release by PorkP was obtained than by ChickP at each concentration. PorkP produced more than a 4-fold increase, whereas ChickP produced nearly 2-fold increase in CCK release over BconP at $5 \mathrm{mg} / \mathrm{ml}$ peptide concentration, and CCK release by PorkP was more than 2-fold higher than that by ChickP at this concentration.

Food intake studies with PorkP and ChickP in rats

After orogastric administration of the peptone preloads, $50 \mathrm{mg}$ ( $1 \mathrm{ml}$ of $50 \mathrm{~g} / \mathrm{l}$ ) PorkP preload markedly suppressed 60-min food intake as compared to that after water preload $(6.41 \pm 0.52$ vs. $7.53 \pm 0.71, P<0.05$, $n=11$ ) (Fig. 2A). The positive control BconP preload also produced similar suppression in the 60-min food intake with the PorkP preload. In contrast, $50 \mathrm{mg}(1 \mathrm{ml}$ of $50 \mathrm{~g} / \mathrm{l}$ ) ChickP did not affect the 60 -min food intake.

The dose-response effect of PorkP on food intake was investigated with a different group of rats as is shown in Fig. 2B. PorkP preload shows a dose-dependent effect on 60-min food intake, with a significant reduction at $50 \mathrm{mg}(1 \mathrm{ml}$ of $50 \mathrm{~g} / \mathrm{l})$ as compared to water preload. Also, PorkP preload inhibited the $60-\mathrm{min}$ food intake similarly to BconP preload.

\section{Discussion}

The present study was designed to find new appetite suppressing peptides associated with CCK release by examining their ability to (1) bind with rat small 

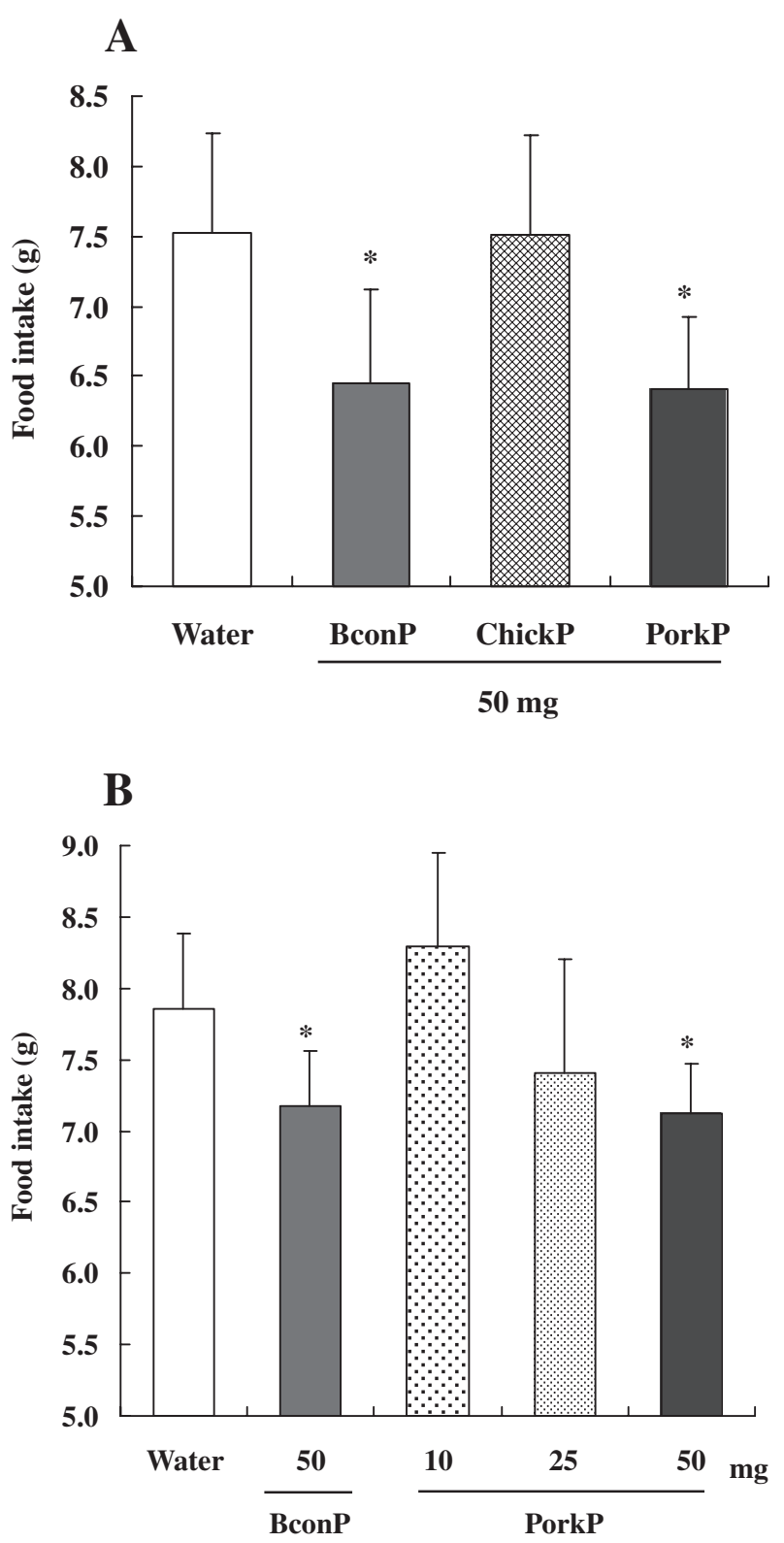

Fig. 2. Food Intake after Orogastric Preloads of Peptone in Rats.

A, Food intake for $60 \mathrm{~min}$ after $50 \mathrm{mg}$ BconP ( $\beta$-conglycinin peptone), ChickP (Chicken peptone) and PorkP (Pork peptone) preloads. Values are means $\pm \mathrm{SEM}, n=11 .{ }^{*}$ Significantly different from rats with water preload at that time $(P<0.05)$. B, Food intake for $60 \mathrm{~min}$ after $50 \mathrm{mg}$ BconP and 10, 25, and $50 \mathrm{mg}$ PorkP preloads. Values are means \pm SEM, $n=8$. ${ }^{*}$ Significantly different from rats with water preload at that time $(P<0.05)$.

intestinal BBM, (2) show CCK-releasing activity from enteroendocrine STC-1 cells, and (3) induce satiety in intact rats with orogastric administration.

ChickP, PorkP, and BeefP showed strong binding to the solubilized components of rat small intestinal BBM among the peptones tested (Table 2). From this screening, we selected two peptones, ChickP and PorkP, showing the strongest binding, for CCK secretion studies. PorkP stimulated CCK release from enderoendocrine cell line STC-1 in a dose-dependent manner ever greater than that produced by ChickP or BconP at every concentration (Fig. 1). Orogastric administration of PorkP, but not ChickP preload, inhibited $60 \mathrm{~min}$ food intake dose-dependently in food-deprived rats, and the inhibitory effect of this peptide mimicked that of the effect of BconP preload (Fig. 2A, B). These results indicate that PorkP is a potent suppressor of food intake via $\mathrm{CCK}$ release, and suggest that the binding of PorkP to the rat small intestinal $\mathrm{BBM}$ is associated with the release of CCK for subsequent reduction in food intake. This is the first report that PorkP suppressed food intake correspondingly with the binding to rat small intestinal $\mathrm{BBM}$ and CCK release from enteroendocrine cells.

In rats, it is thought that dietary protein-induced CCK release from the intestine is regulated by certain endogenous, trypsin sensitive CCK-releasing peptides, ${ }^{1,9)}$ but we and other researchers have found that certain kinds of dietary protein hydrolysates react directly on the small intestinal CCK-producing cells to release the hormone. ${ }^{10,13,14,19)}$ The finding from CCK secretion study that PorkP, ChickP, and BconP released CCK with direct application to STC-1 cells also supports the previous findings. Conversely, it has been shown that endogenous CCK-releasing peptides also stimulate CCK release from STC-1 cells. ${ }^{18)}$ Therefore, despite speculation on a direct interaction of the peptide with CCKproducing cells, we argue another possibility: that peptone also interacts with endogenous CCK-releasing peptide-producing cells to release CCK. In our binding studies, we used whole BBM of rat small intestinal mucosal cells consisting of several kinds of cells, including I-cells. Possibly, the binding activities of peptones also include binding to cells producing the endogenous CCK-releasing peptides.

Our previous study showed that specific peptide structures in BconP are involved in the induction of satiety through CCK release. ${ }^{19)}$ The result of the present study also shows that $10 \mathrm{~min}$ of pepsin digestion might unmask a putative peptide structure(s) of pork meat, and that the highly bio-active pork peptides might induce greater CCK release than BconP from enteroendocrine cells (Fig. 1). But results from food intake studies show that the suppressive effect of PorkP with the threshold dose, viz., $50 \mathrm{mg}$ peptone/rat, is similar to that of BconP at the same dose. We and other researchers have shown that CCK-induced suppression in food intake is associated at least in part with inhibition of gastric emptying. ${ }^{14,22)}$ A possible explanation is that highly bio-active peptides in PorkP released higher amounts of CCK initially, which potently inhibited gastric emptying more than BconP did. Thus, the amount of active peptides in PorkP reaching CCK-producing cells in the small intestine decreased with the delay in gastric emptying. We measured food intake for $60 \mathrm{~min}$. Prolonged release of CCK might be necessary to suppress food intake during this term. This is a possible reason PorkP produced a comparable inhibition to BconP on food intake, even though PorkP has a higher activity to 
release CCK than BconP. We have done experiments using surgically operated rats with duodenal cannula to evaluate the effect of BconP on food intake. ${ }^{14,19)}$ In the present study, we administered peptone preloads into the stomach, not the duodenum, using intact rats without surgical operations to evaluate the effect of peptide under more physiological conditions. The role of PorkP in gastric emptying ought to be clarified in further studies. The structures of the so-called putative bioactive peptide(s) in PorkP must also be characterized.

Since PorkP and ChickP had very high binding activities among the peptones tested, and both peptones stimulated CCK release from STC-1 cells, we provided food intake studies for these two peptones to determine whether they can induce satiety in rats. ChickP had a similar BBM binding activity to the other peptones tested with a still higher CCK release than BconP, but no suppressive effect on 60-min food intake was observed. Previously we found that the active peptide $\beta$ 51-63 in BconP is resistant to trypsin, which may allow this peptide to reach the distal small intestine, ${ }^{19)}$ and no remarkable degradation of ChickP after longer digestion with pepsin or pancreatin was observed in the present study (data not shown). Possibly BconP, but not ChickP, releases other anorectic gut hormones in the distal small intestine. Whether ChickP in the luminal environment requires a synergistic response with other gut hormones to suppress food intake should be clarified in future studies. Also, a higher binding activity to the rat BBM, but a lesser stimulation in CCK release from STC-1 cells, was observed with ChickP than with PorkP. There is a possibility that ChickP formed a strong bond to the rat BBM interacting with intestinal cells other than CCK-producing cells, as mentioned above.

In conclusion, orogastric preload of PorkP suppressed food intake in rats. Also, we found that PorkP stimulated CCK secretion from murine enteroendocrine STC-1 cells at higher levels than BconP, and that this stimulation was associated with the binding of the peptone to rat small intestinal BBM. These results suggest overall that PorkP might have a much higher bio-active peptide(s) responsible for an ever pronounced $\mathrm{CCK}$ release and suppression of appetite in rats.

\section{References}

1) Liddle, R. A., Cholecystokinin cells. Annu. Rev. Physiol., 59, 221-242 (1997).

2) Reidelberger, R. D., Cholecystokinin and control of food intake. J. Nutr., 124, 1327S-1333S (1994).

3) Baile, C. A., McLaughlin, C. L., and Della-Fera, M. A., Role of cholecystokinin and opioid peptides in control of food intake. Physiol. Rev., 66, 172-234 (1986).

4) Reidelberger, R. D., Heimann, D., Kelsey, L., and Hulce, M., Effects of peripheral CCK receptor blockade on feeding responses to duodenal nutrient infusions in rats. Am. J. Physiol. Regul. Integr. Comp. Physiol., 284, R389-398 (2003).

5) Reidelberger, R. D., Hernandez, J., Fritzsch, B., and
Hulce, M., Abdominal vagal mediation of the satiety effects of CCK in rats. Am. J. Physiol. Regul. Integr. Comp. Physiol., 286, R1005-1012 (2004).

6) Trigazis, L., Orttmann, A., and Anderson, G. H., Effect of a cholecystokinin-A receptor blocker on proteininduced food intake suppression in rats. Am. J. Physiol., 272, R1826-R1833 (1997).

7) Liddle, R. A., Green, G. M., Conrad, C. K., and Williams, J. A., Proteins but not amino acids, carbohydrates, or fats stimulate cholecystokinin secretion in the rat. Am. J. Physiol., 251, G243-G248 (1986).

8) Lewis, L. D., and Williams, J. A., Regulation of cholecystokinin secretion by food, hormones, and neural pathways in the rat. Am. J. Physiol., 258, G512-G518 (1990).

9) Liddle, R. A., Regulation of cholecystokinin secretion by intraluminal releasing factors. Am. J. Physiol., 269, G319-G327 (1995).

10) Beucher, S., Levenez, F., Yvon, M., and Corring, T., Effects of gastric digestive products from casein on CCK release by intestinal cells in rat. J. Nutr. Biochem., 5, 578-584 (1994).

11) Cordier-Bussat, M., Bernard, C., Haouche, S., Roche, C., Abello, J., Chayvialle, J. A., and Cuber, J. C., Peptones stimulate cholecystokinin secretion and gene transcription in the intestinal cell line STC-1. Endocrinology, 138, 1137-1144 (1997).

12) Nemoz-Gaillard, E., Bernard, C., Abello, J., Cordier-Bussat, M., Chayvialle, J. A., and Cuber, J. C., Regulation of cholecystokinin secretion by peptones and peptidomimetic antibiotics in STC-1 cells. Endocrinology, 139, 932-938 (1998).

13) Nishi, T., Hara, H., Hira, T., and Tomita, F., Dietary protein peptic hydrolysates stimulate cholecystokinin release via direct sensing by rat intestinal mucosal cells. Exp. Biol. Med., 226, 1031-1036 (2001).

14) Nishi, T., Hara, H., and Tomita, F., Soybean $\beta$ conglycinin peptone suppresses food intake and gastric emptying by increasing plasma cholecystokinin levels in rats. J. Nutr., 133, 352-357 (2003a).

15) Hira, T., Hara, H., and Tomita, F., Characterization of binding between the rat small intestinal brush-border membrane and dietary proteins in the sensory mechanism of luminal dietary proteins. Biosci. Biotechnol. Biochem., 65, 1007-1015 (2001).

16) Liddle, R. A., Misukonis, M. A., Pacy, L., and Balber, A. E., Cholecystokinin cells purified by fluorescence activated cell sorting respond to monitor peptide with an increase in intracellular calcium. Proc. Natl. Acad. Sci. USA, 89, 5147-5151 (1992).

17) Chang, C. H., Chey, W. Y., Sun, Q., Leiter, A., and Chang, T. M., Characterization of the release of cholecystokinin from a murine neuroendocrine tumor cell line, STC-1. Biochim. Biophys. Acta, 1221, 339-347 (1994).

18) Wang, Y., Prpic, V., Green, G. M., Reeve, J. R., and Liddle, R. A., Luminal CCK-releasing factor stimulates CCK release from human intestinal endocrine and STC1 cells. Am. J. Physiol., 282, G16-G22 (2002).

19) Nishi, T., Hara, H., and Tomita, F., The soybean $\beta$ conglycinin $\beta$ 51-63 fragment suppresses appetite by stimulating cholecystokinin release in rats. J. Nutr., 133, 2537-2542 (2003b). 
20) Lowry, O. H., Rosebrough, N. J., Farr, A. L., and Randall, R. J., Protein measurement with the Folin phenol reagent. J. Biol. Chem., 193, 265-275 (1951).

21) Rindi, G., Grant, S. G., Yiangou, Y., Ghatei, M. A., Bloom, S. R., Bautch, V. L., Solcia, E., and Polak, J. M., Development of neuroendocrine tumors in the gastrointestinal tract of transgenic mice: heterogeneity of hormone expression. Am. J. Pathol., 136, 1349-1363 (1990).
22) Moran, T. H., and McHuch, P. R., Cholecystokinin suppresses food intake by inhibiting gastric emptying. Am. J. Physiol., 242, R491-R497 (1982).

23) Reeves, P. G., Nielsen, F. H., and Fahey, G. J., AIN-93 purified diets for laboratory rodents: final report of the American Institute of Nutrition ad hoc writing committee on the reformulation of the AIN-76A rodent diet. J. Nutr., 123, 1939-1951 (1993). 